\title{
An extended laboratory for behavioral neuroscience: A review of Classical Conditioning (third edition)
}

\author{
MICHAEL GABRIEL \\ University of Illinois, Champaign, Illinois
}

Classical Conditioning (3rd ed.)

Edited by I. Gormezano, W. F. Prokasy, and R. F. Thompson. 1987, Erlbaum. 419 pages. \$49.95.

There is much discussion these days about the importance of interaction between the disciplines of neuroscience and psychology. To summarize the argument, it seems that many aspects of the mainstream work in these fields have reached a point of diminishing returns. In several areas of neuroscience (e.g., connectional neuroanatomy, basic neurophysiology, brain distribution of neurochemicals), the important discoveries seem to have been made. Activity in these areas has reached asymptote and is now on the wane. What remains is a plethora of descriptive information and a paucity of information about the functional relevance of what is described. Interdisciplinary studies are needed to provide functional interpretations of the descriptive data that have been obtained.

Experimental-cognitive psychology has seemed for some time now to be beset by the problem of how to define progress. Newer models replace older ones, but neither the old nor the new are intellectually compelling in the way that models of physical and chemical processes have been. There is a feeling of reinvention of the wheel as new principles recapitulate older ones, using slightly different terminology. Some work seems to come unsettlingly close to what physicist and Nobel Laureate Richard P. Feynman (1977) called "cargo-cult science," with reference to the ritualistic ceremonial activities of certain South Sea islanders, whose beliefs were reinforced during World War II by the frequent descent of cargoladen aircraft. The allusion here is to the persistence of theories in psychology that do not stand up to empirical tests. The potential benefit to psychology of interdisciplinary work lies in the weeding out of theoretical ephemera that could be accomplished by the application of neurological constraints.

The field of animal conditioning and learning, especially, would seem to be able to reap substantial benefits from interdisciplinary research. To gain such benefits,

Michael Gabriel's address is Department of Psychology, University of Illinois, $603 \mathrm{E}$. Daniel St., Champaign, IL 61820. however, it is not sufficient for researchers in that area to merely use both neural and behavioral measurements in their experiments. In addition, a kind of bootstrapping is required, wherein parallel psychological and neural analyses continually guide each other. For example, try to imagine a neural model of rat radial-arm maze performance that did not address neural mediation of the role of extramaze stimuli. Similarly, any neural process that is believed to be essential for the performance of some behavior (such as the activity of cerebellar deep nuclear neurons for classical conditioning of the nictitating membrane response in rabbits) ought to have a counterpart in the psychological theory of that behavior. Thus, it is important for behavioral neuroscientists to use adequate theoretical models of the target behavioral phenomena to guide state-of-the-art neuroanalytic studies leading to a description of the neurological causation of the behavior. It is very likely that the process of working out the neural causation will foster major revisions or complete redefinitions of the psychological models. The ultimate aim is interchangeability of the terms and parameters of the two kinds of models.

Of course, these things are easy to say. The hard part is getting the work done. How is this to happen? Where are the scientists trained in the history, methods, theories, and customs that are essential for the conduct of neuroand behavior-analytic studies of animal conditioning and learning processes? Although a goodly number of scientists know both traditions well and are capable of both methodologies, it is almost impossible to obtain resources sufficient to permit meaningful pursuit of both lines of work. Thus, only a precious few single investigators (SIs) are succeeding in actually performing this very difficult task.

The practical alternative to the SI model would seem to be a division-of-labor (DOL) model wherein individuals specialize in one or the other approach, with the hope that the critical interplay needed for progress will occur as a result of between-laboratory interactions. The big problem with this is standardization. People doing a neurological analysis of behavioral learning have to be sure that they are studying exactly the same behavioral phenomenon being subjected to behavioral and theoretical analyses else- 
where. Such uniformity is hard to achieve for several reasons, including the notorious sensitivity of conditioned responses to subtle environmental and procedural variations, and the special laboratory arrangements often needed for neural analyses but not in the behavioral lab. These problems are serious blocks to the success of the DOL model. Indeed, the DOL model essentially represents the status quo, and it may not be too pessimistic to say that the status quo is not working very well. Thus, to summarize the dilemma, neither the SI nor the DOL model is particularly good as a cultivator of the interdisciplinary approach that is the essence of behavioral neuroscience.

The work reported in the third edition of Classical Conditioning suggests a provocative solution to this problem. This book contains 14 chapters, most of which deal in one way or another with the analysis of classical conditioning of the eyeblink and nictitating membrane (NM) responses in rabbits. Nine of the chapters deal with the neural bases of the conditioned and unconditioned responses; 4 deal with purely behavioral analyses of this paradigm, and 1 (chap. 2, by N. Schneiderman and his colleagues) reviews extensive analyses of the neural subtrates of cardiovascular conditioning in rabbits.

The chapters are well written, and the work reported is of a very high quality. However, I am impressed not only by the excellence of the individual contributions, but also by the unique and possibly unprecedented socioscientific character of this work. Globally considered, the contents of Classical Conditioning are unique with regard to the numbers of scientists engaged simultaneously in the analysis of a single conditioning paradigm, the inclusion of sophisticated and in-depth neural as well as behavioral analyses, the widespread consensus among participants regarding the procedures used for controlling the behavior, and the remarkable replicability of both neural and behavioral phenomena across laboratories when agreed-upon parameters have been adopted. (The last two points are discussed by M. Patterson in the book's first chapter, which sets forth virtually all one needs to know about rabbits, and describes the conditioning procedures and apparatus employed in the majority of the book's remaining chapters. This throughgoing chapter is a sine qua non for investigators who are setting up a rabbit conditioning laboratory for the first time.) Additionally, there is an unprecedented and almost familial closeness among the participants in this research, inasmuch as virtually all of them are students or students of students either of the originator of the paradigm (I. Gormezano) or of the originator of the neurological analysis of the paradigm ( $R$. F. Thompson).

The features of the work described in Classical Conditioning suggest that the group of involved participants and facilities form, in effect, an extended laboratory (EL). That is, the resulting consensus and uniformity of practice, while not as great as can be achieved in the SI case, are substantially greater than is typically found when the DOL type of organization is operating. The effect is much like having one laboratory spread over 20 or so locations throughout the world.
I would like to suggest that this arrangement may approximate an optimal one for achieving the goals of behavioral neuroscience-optimal in the sense that it should permit cross-fertilization between the behavioral and the neural levels of analysis while also being within reach of the capacities and resources of individual investigators. The principal reason for this, familial closeness notwithstanding, is the uniformity of procedures and results that appears to be achievable across laboratories.

Of course, the proof is in the pudding. The question is, has the rabbit EL yielded the desired crossfertilizations? The answer would appear to be that, given the relative youth of the rabbit EL (by my reckoning, it is about 8 years old), some very promising beginnings have been made. For example, both the now-classic findings demonstrating the very rapid electrophysiological changes that precede the first signs of behavioral acquisition (T. Berger, chap. 7) and the more recent findings indicating that there may exist a pharmacologically distinct (opiate-sensitive) learning system underlying the early stages of behavioral acquisition ( $R$. F. Thompson et al., chap. 14) quite nicely fit the elegant theoretical and mathematical analysis of the rabbit conditioning paradigm proposed by W. F. Prokasy (chap. 11). Berry, Weisz, and Mamounas (chap. 9) report the intriguing finding of dramatic theta-like conditioned stimulus(CS)-elicited hippocampal cellular discharges in a jaw-movement (appetitive) conditioning paradigm, but no such discharges in the standard aversive NM-conditioning paradigm. This asymmetry of the electrophysiological data may provide important clues to the neural factors that distinguish aversive and appetitive conditioning processes. This, in turn, could illuminate the interesting behavioral asymmetry reported by M. Scavio (chap. 12), who showed that there is negative transfer of training when appetitive conditioning is administered following aversive conditioning with the same CS, but that positive transfer is found when the order of these paradigms is reversed.

An EL could fail if the participants closed ranks when disagreements and/or empirical discrepancies arose. However, history has indicated that participants in the rabbit EL are independent self-determining individuals. For example, Berthier, Desmond, and Moore (chap. 10) and Thompson et al. (chap. 14) report that a certain discrete unilateral lesion abolishes the conditioned response (CR) on the side of the lesion but that the unconditioned response remains intact. However, the site of the lesion yielding these effects is, in each case, different. For Thompson et al., it is the deep cerebellar interpositus nucleus, but for Berthier et al., it is the supratrigeminal reticular formation. Each result, taken singly, could be interpreted as indicating that the lesions interfere with circuitry that is essential for CR mediation, and that the lesion site may be at or very near the locus of the critical neural plasticity underlying CR acquisition. It is clear, of course, that both sites cannot simultaneously be parts of independent circuits essential for the CR, or the single lesions in either site would not be effective. Although future work may indicate that these areas are directly interconnected, and thus that the lesions disrupt different points 
in a single pathway, there are presently no compelling data to verify such an interpretation. Whatever the resolution of this issue, the issue itself can serve to illustrate an important advantage of EL organization. Discrepant findings are much more likely to be detected and much less likely to be overlooked in ELs, which have a strong autoevaluation mechanism, than in SI and DOL organizations. Clearly, the understanding of the neural organization underlying $C R$ acquisition and performance will be advanced when this discrepancy is resolved. The autoevaluative process also operates in a positive way. Research taking two different, essentially independent paths within the rabbit EL (reviewed by Cegavske, Harrison, and Torigoe, chap. 3, and by Berthier et al., chap. 10) seem to have converged on the delineation of the circuitry essential for mediation of the unconditioned response.

An enormous amount of research has been directed toward the hippocampus and related limbic structures in relation to the neural mediation of learning and memory processes. Most of this work is intrahippocampal: it does not deal with the interactions of the hippocampal system and the rest of the brain. The work of the rabbit EL has been unique in showing that it can elucidate the interactions of the hippocampal system with the lower brain circuitry directly concerned with the NM and eyeblink CRs. Studies reviewed by Berger and Weisz (chap. 8), P. Solomon (chap. 5), and R. Salafia (chap. 7) indicate that damage to the hippocampal system does not affect CR acquisition, but that such damage eliminates or attenuates the phenomena of reversal learning, latent inhibition, and the blocking effect. Other evidence strongly supports the conclusion that cerebellar and brainstem circuits constitute the essential substrates of CR acquisition (see the extensive and elegant analyses reported by Thompson et al., chap. 14, and Berthier et al., chap. 10). The implication is clear that hippocampal modulatory control of the NM and eyeblink CR is achieved by way of circuitry linking the hippocampal formation with the cerebellar and brainstem areas that are more fundamentally in control of the CR. Thus, it would seem that the rabbit EL is poised to make a most important contribution, that is, to provide a detailed account of the circuit-level interactions of the hippocampal system with the cerebellar and brainstem circuitry responsible for basic CR acquisition. Berger has already begun this work with studies of the hippocampal system that extend to the cerebellum via the retrosplenial cortex and the pontine nuclei.

It is important to note the findings reviewed in Chapters 5 and 7 concerning the overproduction of CRs in rabbits with hippocampal damage during performance in paradigms such as reversal learning, blocking, and latent inhibition. These findings are fundamentally similar to those dating back to the 1950 s in indicating that hippocampal damage induces $C R$ overproduction in a wide array of operant behaviors (e.g., discrimination reversal learning in several appetitive paradigms, differential reinforcement of low rate performance, extinction of approach be- havior, and others). These earlier studies and the recent ones in the rabbit EL can be interpreted to indicate that animals with hippocampal damage fail to suppress learned behavior when they experience novel or unexpected stimuli and/or stimulus contingencies in the training environment. These results raise the possibility that future discoveries made in ELs regarding the circuitry that enables the hippocampal system to modulate NM and eyeblink CRs in rabbits will have generality with respect to a large class of behavior, not just rabbit CRs.

Are there any shortcomings of ELs in general and of the rabbit EL in particular? One possibility is a kind of myopia, a common problem of inbreeding. One example is the absence of contact between some of the rabbit EL work and the more general work on the neuropsychology of the hippocampus, which includes the work on the disinhibitory effects of hippocampal lesions mentioned above. If such contact had been made, the rabbit EL group could possibly have avoided the erroneous conclusion drawn early on-and repeated in some of the chapters in Classical Conditioning - that the hippocampal system represents an essential substrate of the behavioral CR, rather than being a source of CR modulation. Also essentially ignored in citation lists of the rabbit EL is work, ongoing since 1974, involving the use of an avoidance conditioning paradigm with rabbits to study the interaction of the hippocampal system with certain subcortical areas (i.e., the anterior and medial dorsal "limbic" nuclei of thalamus) that appear to be essential substrates of the CR. These studies of avoidance have fostered a theoretical working model of the neural circuitry for the inhibitory modulation of the CR-driving thalamic substrates (Gabriel, Foster, Orona, Saltwick, \& Stanton, 1980; Gabriel, Sparenborg, \& Stolar, 1986). Many interesting possibilities emerge from a comparison of the results of the rabbit EL studies and these studies of avoidance. An obvious example is the idea of a central neural organization for conditioning wherein separate systems exist for CR generation (e.g., the limbic nuclei for avoidance and the cerebellar interpositus nucleus for the NM CR), even though these separate systems are all subjected to a similar form of hippocampal inhibitory modulation.

\section{REFERENCES}

Feynman, R. P. (1985). "Surely you are joking Mr. Feynman!" Adventures of a curious character. New York: Bantam.

Gabriel, M., Foster, K., Orona, E., Saltwick, S., \& Stanton, M. (1980). Neuronal activity of cingulate cortex, anteroventral thalamus and hippocampal formation in discriminative conditioning: Encoding and extraction of the significance of conditional stimuli. In J. Sprague \& A. N. Epstein (Eds.), Progress in psychobiology and physiological psychology (Vol. 9, pp. 126-223). New York: Academic Press.

Gabriel, M., Sparenborg, S., \& Stolar, N. (1986). An executive function of the hippocampus: Pathway selection for anterior thalamic neuronal significance code. In R. L. Isaacson \& K. H. Pribram (Eds.), The hippocampus (Vol. 4, pp. 1-35). New York: Plenum Press.

(Manuscript received February 2, 1988; accepted for publication February 10, 1988.) 